\title{
Disorder broadening of even denominator fractional quantum Hall states in the presence of a short-range alloy potential
}

\author{
E. Kleinbaum ${ }^{1}$, Hongxi Li ${ }^{1}$, N. Deng ${ }^{1}$, G.C. Gardner ${ }^{1,2}$, M.J. Manfra ${ }^{1,2,3}$, and G.A. Csáthy ${ }^{1,2}$ \\ 1 Department of Physics, Purdue University, \\ West Lafayette, IN 47907, USA \\ 2 Birck Nanotechnology Center, \\ Purdue University, West Lafayette, IN 47907, USA \\ 3 School of Materials Engineering and School of Electrical and Computer Engineering, \\ Purdue University, West Lafayette, IN 47907, USA
}

(Dated: September 7, 2020)

\begin{abstract}
We study energy gaps of the $\nu=7 / 2$ and $\nu=5 / 2$ fractional quantum Hall states in a series of twodimensional electron gases containing alloy disorder. We found that gaps at these two filling factors have the same suppression rate with alloy disorder. The dimensionless intrinsic gaps in our alloy samples obtained from the model proposed by Morf and d'Ambrumenil are consistent with numerical results, but are larger than those obtained from experiments on pristine samples published in the literature. The disorder broadening parameter has large uncertainties. However, a modified analysis relying on shared intrinsic gaps yields consistent results for both the $\nu=5 / 2$ and $7 / 2$ fractional quantum Hall states and establishes a linear relationship between the disorder broadening parameter and alloy concentration. Furthermore, we find that we can separate contributions to the disorder broadening of the long-range and short-range scattering.
\end{abstract}

Disorder is one of the least understood factors impacting many-body ground states, such as the ones forming in the two-dimensional electron gas (2DEG). As disorder levels in 2DEGs in GaAs/AlGaAs heterostructures are reduced, there is an increasing number of FQHSs developing 1 ] The influence of disorder on other manyelectron states, such as different types of electron solids, is more intricate ${ }^{8}$.

The importance of quantifying disorder effects on the energy gap of FQHSs was recognized early on and played a role in establishing the Laughlin nature of the $\nu=1 / 3$ FQHS 4 7. These efforts yielded a simple phenomenological model that relates $\Delta^{\text {meas }}$, the measured energy gap, to $\Delta^{i n t}$, the gap obtained from numerical simulations in disorder-free models ${ }^{4} \cdot 7 . \Delta^{i n t}$ is referred to as the intrinsic gap. According to this phenomenological model, the measured energy gap $\Delta^{\text {meas }}$ is reduced as compared to the intrinsic gap $\Delta^{i n t}$ by the so called disorder broadening parameter $\Gamma$ :

$$
\Delta^{\text {meas }}=\Delta^{\text {int }}-\Gamma .
$$

More recent theoretical efforts sought understanding of the disorder-driven collapse of FQHS $\frac{11112}{}$ and of the impact of disorder on quantum entanglement 13 15.

Even denominator FQHSs, such as the ones developing at $\nu=5 / 2$ and $\nu=7 / 2$ in 2DEGs in the GaAs/AlGaAs system $^{16}-19$, are also affected by disorder 20,30 . These FQHSs continue to attract interest because of their possible non-Abelian excitations ${ }^{31} 33$. Within the framework of the phenomenological model for the energy gaps presented above, in order to extract the intrinsic gap from measurements one must independently obtain both $\Delta^{\text {meas }}$ and $\Gamma$ from the measured data. This is clearly not possible from a single measurement, say from the knowledge of $\Delta_{5 / 2}^{\text {meas }}$, the measured energy gap of the $\nu=5 / 2$
FQHS. Instead, Morf and d'Ambrumeni ${ }^{34}$ proposed an analysis of gaps based on the measurement of two independent quantities, the gaps measured at particle-hole conjugated filling factors $\nu=5 / 2$ and $\nu=7 / 2$. Such an analysis was first applied to 2DEGs of the highest available mobility ${ }^{34}$, henceforth referred to as pristine 2DEGs. The extracted intrinsic gap values were in reasonable agreement with numerical results both in high density samples ${ }^{2554}$, with electron density close to $3 \times 10^{11} \mathrm{~cm}^{-2}$, as well as in samples of reduced density 2 , as low as $8.3 \times 10^{10} \mathrm{~cm}^{-2}$.

The analysis of Morf and d'Ambrumeni ${ }^{34}$ of the even denominator FQHSs remains important since efforts to relate energy gaps and disorder broadening parameters of these states to lifetime parameters failed in pristine samples. Indeed, it was found that the measured energy gap of the $\nu=5 / 2 \mathrm{FQHS}$ does not correlate in an obvious way with either the transport lifetime $\mathrm{E}^{20} \sqrt{28}$ or the

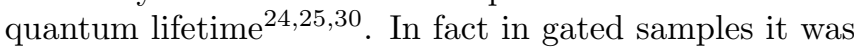
found that the quantum lifetime is approximately constant over the density range at which the energy gap at $\nu=5 / 2$ decreased from its largest value to zerd $24 \mid 30$. The lack of correlation between the energy gap and lifetime parameters is not surprising since, in contrast to the energy gap at $\nu=5 / 2$, both the transport and the quantum lifetimes are measured at or near zero magnetic field, in a regime in which single-electron descriptions work well.

Besides pristine samples, the energy gap of the $\nu=5 / 2$ FQHS was also studied in a series of samples with short range scattering centers deliberately introduced during the Molecular Beam Epitaxy (MBE) growth process ${ }^{28}$. Since these short range scattering centers were $\mathrm{Al}$ atoms added to the GaAs channe ${ }^{35 \mid 36}$, we will refer to these samples as alloy samples. Since energy gap measurements at $\nu=7 / 2$ in alloy samples were not available, 
the analysis of Morf and d'Ambrumenil for the even denominator FQHSs in alloy samples so far could not be performed.

In this Article we report energy gap measurements for the $\nu=7 / 2$ FQHS in GaAs/AlGaAs based samples with alloy disorder. We found that gaps at $\nu=7 / 2$ and $\nu=5 / 2$ have the same suppression rate with alloy disorder. These samples with alloy disorder provided an opportunity to examine the model put forth by Morf and d'Ambrumenil ${ }^{34}$ in the presence of a short-rage scattering potential. We found that this model yields intrinsic energy gaps consistent with numerical results, albeit larger than those obtained from pristine samples published in the literature. The disorder broadening parameter extracted using this model had a significant scatter that resulted in an unreasonable dependence on the alloy content of samples. However, the additional information of shared dimensionless energy gap can be exploited to determine $\Gamma$ and its alloy content dependence. This analysis allowed us to separate contributions to the disorder broadening due to alloy scattering and other scattering mechanisms. Our results highlight the contrast between effects of short-range and long-range scattering potentials on the even denominator FQHSs, further understanding of the energy gaps of these states, and open the door for future studies of other types of limiting disorder, such as charged impurity disorder.

Samples used in this study are the same as those in Ref. ${ }^{28}$. They are $30 \mathrm{~nm}$ quantum well samples of nearly the same electron density, but in which the GaAs channel has $\mathrm{Al}$ atoms added during the MBE growth process

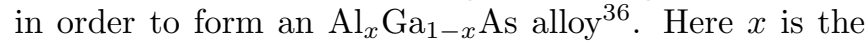
$\mathrm{Al}$ molar fraction which is significantly less than the $\mathrm{Al}$ molar fraction in the confining layers and it therefore does not affect the electronic wave function perpendicular to the plane of the 2DEG. Detailed sample structures and characterization can be found in Ref ${ }^{36}$. Electrical transport measurements were performed at dilution refrigerator temperatures in a van der Pauw geometry, after samples were illuminated with a red light-emitting diode 28 . Samples were mounted in a He-3 immersion cell and the temperature $T$ of the He-3 liquid was monitored by quartz tuning fork viscometry 37 .

Fig. 1 shows the longitudinal magnetoresistance $R_{x x}$ and Hall resistance $R_{x y}$ in the range of filling factors $3<\nu<4$ known as the upper spin branch of the second Landau level. Data are shown for three samples: the pristine sample $x=0$ and two alloy samples with $x=0.00057$ and $x=0.00075$. Due to its high mobility of $20 \times 10^{6} \mathrm{~cm}^{2} / \mathrm{Vs}$, the pristine sample exhibits all known ground states. The most prominent FQHS is the one at $\nu=7 / 2$, but there are also others at $\nu=3+1 / 5$, $\nu=3+4 / 5^{19}$ and $\nu=3+1 / 3^{38}$. The high quality of the sample also allows us to observe eight reentrant integer quantum Hall states, ground states associated with electronic bubble phases $\$ 19139$.

An increasing alloy content $x$ has a strong impact on magnetotransport. The FQHSs at $\nu=3+1 / 5$ and $\nu=$

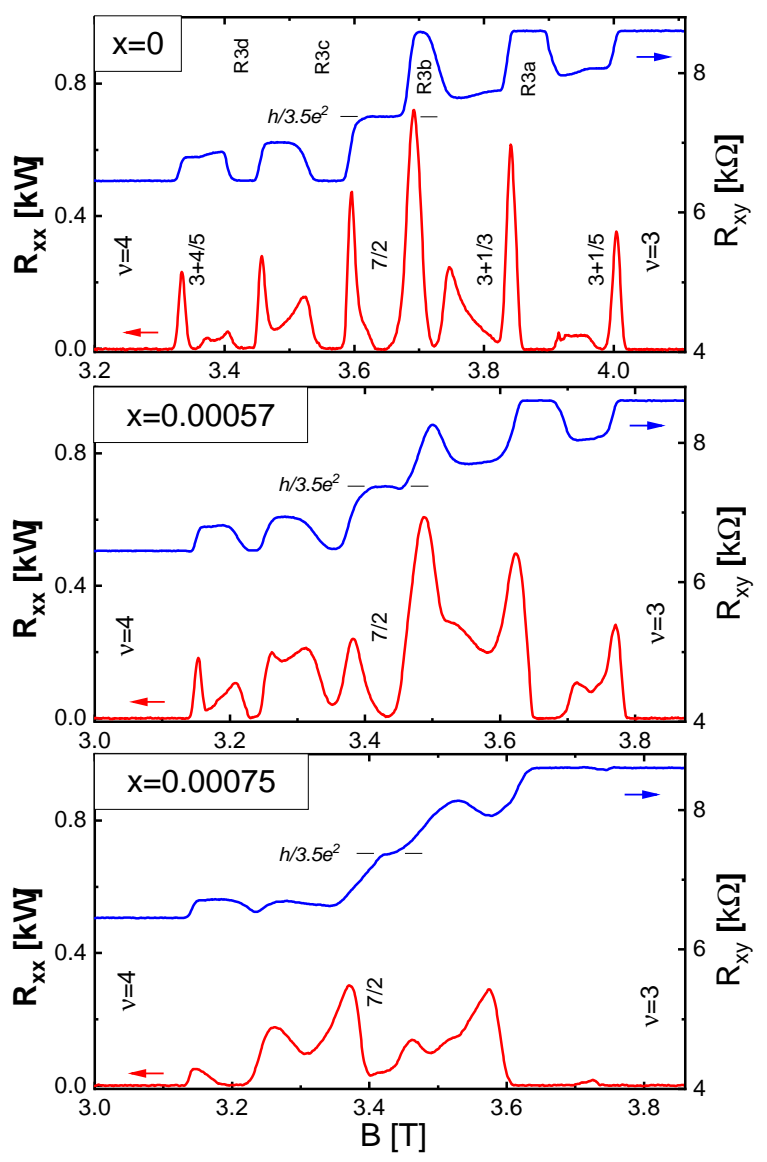

FIG. 1. Magnetoresistance $R_{x x}$ and Hall resistance $R_{x y}$ in samples with $x=0, x=0.00057, x=0.00075$ alloy content in the filling factor range of $3<\nu<4$ measured at $7 \mathrm{mK}$. Numbers indicate the filling factors of various FQHSs; reentrant integer quantum Hall states associated with electronic bubble phases are labeled $R 3 a, R 3 b, R 3 c$ and $R 3 d$.

$3+4 / 5$ significantly weaken and the one at $\nu=3+1 / 3$ is destroyed at the lowest non-zero alloy concentration $x=0.00057$. Similarly, magnetotransport in the bubble phases is impacted. However, as observed in both $R_{x x}$ and $R_{x y}$ data, the FQHS at $\nu=7 / 2$ survives at both $x=0.00057$ and $x=0.00075$, but it is destroyed in the sample with $x=0.0015$ (not shown).

In order to characterize the $\nu=7 / 2$ FQHS, we measure its energy gap. In Fig.2 we show the temperature dependence of the longitudinal magnetoresistance measured at $\nu=7 / 2$ in the three samples in which a FQHS at this filling factor is present. As seen in the Arrhenius plots of Fig.2, $R_{x x}$ of all three samples follows an activated form $R_{x x} \propto \exp \left(-\Delta_{7 / 2}^{\text {meas }} / 2 k_{B} T\right)$ at the lowest temperatures. Energy gaps $\Delta_{7 / 2}^{\text {meas }}$ extracted are $139 \mathrm{mK}, 87 \mathrm{mK}$ and $23 \mathrm{mK}$ at $x=0, x=0.00057$ and $x=0.00075$, respectively.

Similarly to observations at $\nu=5 / 228$, the measured energy gap at $\nu=7 / 2$ is suppressed by an increasing $x$. Consistent with prior knowledge from pris- 


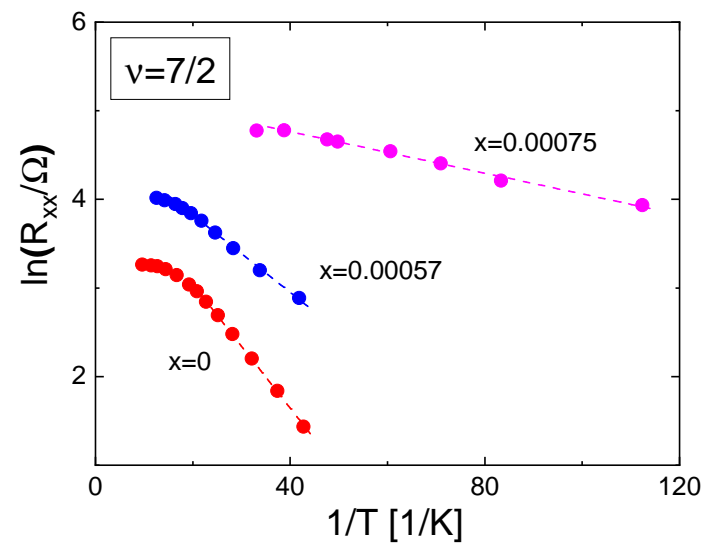

FIG. 2. Arrhenius plots for the $\nu=7 / 2$ FQHS at different Al molar fraction $x$. Dotted lines are linear fits in the activated regime used to extract energy gaps.

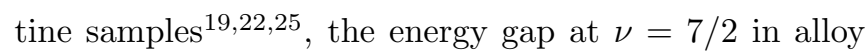
samples is also significantly less than that measured at $\nu=5 / 2$. As seen in Fig.3, energy gaps for both FQHSs exhibit a linear trend with the alloy content $x$; gap suppression rate at both $\nu=5 / 2$ and $\nu=7 / 2$ are similar, with a value of $\delta \Delta^{\text {meas }} / \delta x \approx 0.15 \times 10^{3} \mathrm{~K}$. This behavior indicates that both $\nu=5 / 2$ and $\nu=7 / 2$ FQHSs respond in a similar fasion to alloy disorder.

While the two energy gaps plotted against $x$ in Fig.3 exhibit linear trends, data display significant scatter. Scatter in this figure has two distinct sources: scatter in the energy gap and scatter in $x$, the $\mathrm{Al}$ content of the alloy channel. Scatter in the energy gap is well-known from measurements in pristine samples. For example, after repeated cycling of the sample to room temperature, the measured energy gap is known to have small varition variations. Similarly, variations in the sample state due to the sample illumination procedure are likely present. Systematic errors in the temperature measurement may also contribute to errors in the energy gap. These sources of scatter are often estimated to contribute to about $\pm 10 \%$ error to the gap. In addition, some scatter of data in Fig. 3 can be associated the alloy forming process. Because of the extreemely low amount of $\mathrm{Al}$ in the channel of our alloy samples, the Al effusion cell used for the channel alloy formation is operated in a regime of very low flux ${ }^{36}$. Under these conditions there will be errors in $x$, the $\mathrm{Al}$ content of the channel. While such errors are diffcult to quantify, their presence can be observed as deviations from a linear dependence of the scattering rate versus $x$ in Ref ${ }^{36}$. We attribute to errors in $x$ the correlated scatter of the energy gaps at $\nu=5 / 2$ and $\nu=7 / 2$ present at the three lowest $x$ values in Fig.3.

In the following we analyze the energy gaps of the two even denominator FQHSs using the model proposed by Morf and d'Ambrumeni[34. Since FQHSs are many-body ground states, the intrinsic energy gap scales with the Coulomb energy $\Delta^{i n t}=\delta^{i n t} E_{C}$. Here $E_{C}$ is the Coulomb

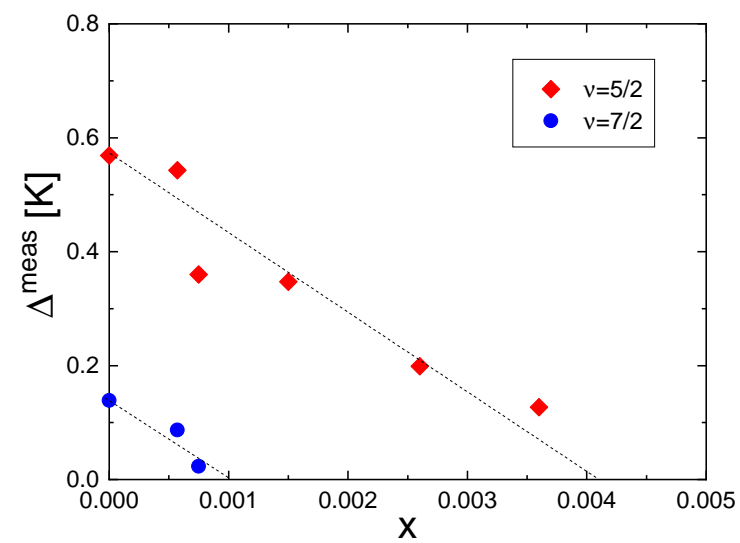

FIG. 3. Dependence of the measured energy gaps at $\nu=5 / 2$ and $\nu=7 / 2$ on the alloy content $x$ of the channel. Energy gaps for the $\nu=5 / 2$ FQHS are from Ref. ${ }^{28}$. Dashed lines are guides to the eye.

energy $E_{C}=e^{2} / 4 \pi \epsilon l_{B}, l_{B}=\sqrt{\hbar / e B}$ is the magnetic length. The dimensionless intrinsic gap $\delta^{\text {int }}$ depends on the type of electronic correlations, the thickness of the 2DEG in a direction perpendicular to the plane of the $2 \mathrm{DEG}^{24 / 40} 46$, and Landau level mixing 24/34/45 49. Morf and d'Ambrumenil assumed that $\delta^{\text {int }}$ is shared for the two even denominator FQHSs therefore, based on Eq.(1), the two energy gaps $\Delta_{5 / 2}^{\text {meas }}$ and $\Delta_{7 / 2}^{\text {meas }}$ both satisfy

$$
\Delta^{\text {meas }}=\delta^{i n t} E_{C}-\Gamma \text {. }
$$

Within this model, the disorder broadening parameter $\Gamma$ is also shared by the $\nu=5 / 2$ and $\nu=7 / 2$ FQHSs and independent measurements of $\Delta_{5 / 2}^{\text {meas }}$ and $\Delta_{7 / 2}^{\text {meas }}$ allow the extraction of the two parameters $\delta^{i n t}$ and $\Gamma$. This is achieved by plotting the two measured gaps against $E_{C}$ and by fitting a line to the two data points 34 ; the slope of this line is $\delta^{i n t}$, whereas the intercept with the vertical scale is $\Gamma$. Such an analysis is shown in Fig.4 for the three alloy samples exhibiting the $\nu=7 / 2$ FQHS and we summarized the parameters of the model in Table.I. $\Delta_{5 / 2}^{\text {meas }}$ used in our analysis are from Ref. ${ }^{28}$.

Analyses following the model of Morf and d'Ambrumenil of the gaps in pristine samples from the literature of electron density close to that of ours yielded $\delta^{\text {int }}=0.014^{34}$ in a sample of $n=3.0 \times 10^{11} \mathrm{~cm}^{-2}$ from

TABLE I. A summary of the dimensionless intrinsic gap $\delta^{\text {int }}$ and disorder broadening parameter $\Gamma$ (in units of $\mathrm{K}$ ) for the $\nu=5 / 2$ and $\nu=7 / 2$ FQHSs in a series of alloy samples.

\begin{tabular}{lllllll}
\hline \hline$x$ & 0 & 0.00057 & 0.00075 & 0.0015 & 0.0026 & 0.0036 \\
\hline$\delta^{\text {int }}$ & 0.024 & 0.026 & 0.020 & - & - & - \\
$\Gamma$ & 2.21 & 2.40 & 1.82 & - & - & - \\
$\tilde{\Gamma}_{5 / 2}$ & 2.20 & 2.23 & 2.41 & 2.42 & 2.57 & 2.64 \\
$\tilde{\Gamma}_{7 / 2}$ & 2.20 & 2.25 & 2.32 & - & - & - \\
\hline \hline
\end{tabular}




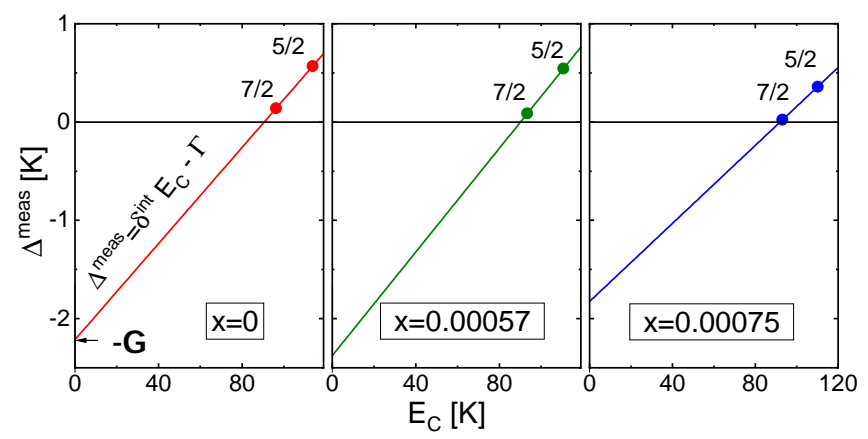

FIG. 4. Energy gaps $\Delta_{5 / 2}^{\text {meas }}$ and $\Delta_{7 / 2}^{\text {meas }}$ plotted against the Coulomb energy $E_{C}$. According ideas put forth in Ref. ${ }^{34}$, the slope of the line through the two data points is $\delta^{i n t}$, whereas the intercept of each line with the vertical scale is the disorder broadening parameter $\Gamma$.

Ref. ${ }^{19}, \delta^{\text {int }}=0.019^{25}$ in a sample of $n=2.78 \times 10^{11} \mathrm{~cm}^{-2}$ from Ref. ${ }^{25}$, and $\delta^{\text {int }}=0.01{ }^{25}$ in a sample of $n=3.0 \times 10^{11} \mathrm{~cm}^{-2}$ from Ref. $\frac{50}{}$. In our pristine sample $x=0$ we find $\delta^{\text {int }}=0.024$, a value significantly larger than those from the literature 25134 . Nonetheless, this value is consistent with numerical results as it is less than $\delta^{\text {int }} \approx 0.0341445151$ and $\delta^{\text {int }} \approx 0.036^{24}$, values obtained in the limit of no Landau level mixing and zero layer thickness, but it is larger than the values $\delta^{i n t}=0.016$, $\delta^{\text {int }}=0.018^{24}$, and $\delta^{\text {int }}=0.016^{48}$ from estimations that include both Landau level mixing and finite layer thickness effects. However, such a consistency can only be considered crude at best because of systematic errors in our experiment discussed earlier and also because the assumption of equal $\delta^{i n t}$ for the $\nu=5 / 2$ and $7 / 2$ FQHSs is only approximate in measurements of samples of fixed density due to the slightly different Landau level mixing parameters at these filling factors.

Since the series of alloy samples were engineered to have the same width of their quantum well and have similar electron densities, their intrinsic gap is expected to be similar. This is because within the phenomenological model embodied by Eqs.(1) alloy disorder factors only into the disorder broadening parameter, and not into the intrinsic gap. Dimensionless intrinsic gaps of our alloy samples listed in Table.I. are reasonably close, within a error of $\pm 13 \%$. The sample with $x=0.00075$ has its $\delta^{\text {int }}$ the farthest from values in the other two samples. We ascribe the scatter of $\delta^{i n t}$ to earlier discussed systematic errors.

As the amount of disorder increases, the measured energy gap is suppressed and the disorder broadening parameter is expected to increase. This trend can be observed for samples with $x=0$ and $x=0.00057$, but it breaks down as $x$ increases to $x=0.00075$. This unphysical non-monotonic dependence of $\Gamma$ on $x$, shown in Fig.5, is likely due to the same errors that led to variations in $\delta^{\text {int }}$. Because of multiple sources of errors, we are not able to disentangle the influence of different sources or error on the disorder broadening parameter. We con-

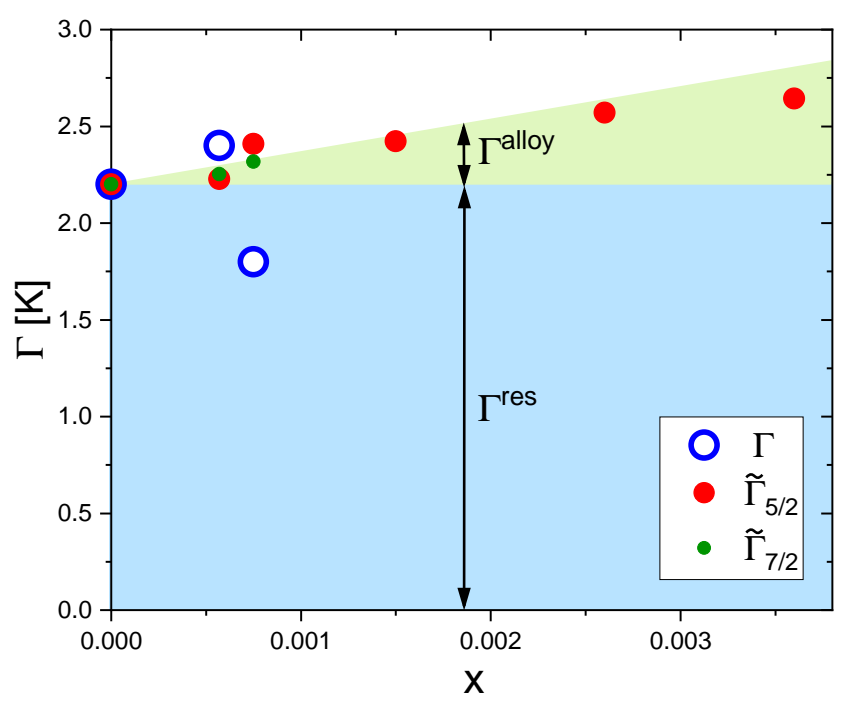

FIG. 5. Comparison of $x$ dependent disorder broadening parameters extracted using different models. Open symbols represent $\Gamma$ extracted according to Ref ${ }^{[34}$ while solid symbols are $\tilde{\Gamma}$ extracted with a fixed intrinsic gap at $\nu=5 / 2$ and $\nu=7 / 2$, as described in the text.

clude that, due to accumulating errors, the model of Morf and d'Ambrumenil does not yield satisfactory disorder broadening parameters in our alloy samples.

For an improved analysis we exploit the property of shared $\delta^{\text {int }}$ in our series of samples. In the following we calculate a modified version of the disorder broadening $\tilde{\Gamma}$ which is still based on Eq.(2), but in which we fix $\delta^{\text {int }}=$ 0.024 , its value in our pristine sample. The parameter $\tilde{\Gamma}=\delta^{\text {int }} E_{C}-\Delta^{\text {meas }}$ can be independently calculated at both $\nu=5 / 2$ and $\nu=7 / 2$. We label these $\tilde{\Gamma}_{5 / 2}$ and $\tilde{\Gamma}_{7 / 2}$, list the obtained values in Table.I., and plot these values against $x$ in Fig.5. We found that both $\tilde{\Gamma}_{5 / 2}$ and $\tilde{\Gamma}_{7 / 2}$, when plotted against $x$, exhibit significantly less scatter than $\Gamma$ and both have an increasing trend with $x$, in agreement with our expectation. Moreover, $\tilde{\Gamma}_{5 / 2}$ and $\tilde{\Gamma}_{7 / 2}$ are very close, with the largest difference of only $\pm 2 \%$. This shows that the modified analysis is self-consistent and it is also consistent with the behavior shown in Fig. 3 of similar $\delta \Delta^{\text {meas }} / \delta x$ slopes at $\nu=5 / 2$ and $\nu=7 / 2$. We conclude that the modified analysis for obtaining the disorder broadening parameter led to a significant improvement in the scatter of the data.

Fig.5 suggests that the disorder broadening parameter $\Gamma$ has two separate contributions: one due to short range disorder scattering centers $\Gamma^{\text {alloy }}$ and another due to the residual scattering $\Gamma^{r e s}$. The latter we associate with long range scattering due to a smoothly varying scattering potential of ionized impurities present in all our samples; $\Gamma^{r e s}$ is independent of the disorder level $x$. We suggest that the disorder broadening admits the following separation: $\Gamma=\Gamma^{r e s}+\Gamma^{\text {alloy }}(x)$. Such a separation of the contributions of ionized impurities and alloy impurities in 
our alloy samples is possible since they are grown under similar conditions, thus share a similar long range scattering potential. We also found that for the range of alloy disorder studied, $\Gamma^{\text {res }} \gg \Gamma^{\text {alloy }}(x)$. This finding quantifies earlier qualitative results, according to which the long range potential due to ionized impurities is more detrimental to the even denominator FQHSs than short range potentials $26 \mid 28$. Our data yields $\Gamma^{\text {alloy }}(x)=0.17 \times 10^{3} x$ in units of K. Finally we note that the $\Gamma=\Gamma^{\text {res }}+\Gamma^{\text {alloy }}(x)$ relationship is reminiscent of Matthiessen's rule for the scattering times: $1 / \tau^{\text {tot }}=1 / \tau^{\text {res }}+1 / \tau^{\text {alloy }}(x)$ found in disorder samples $35 / 36$. Here $\tau^{\text {tot }}$ is the scattering time or mobility lifetime in alloy samples, $\tau^{\text {res }}$ is the scattering time due to the ionized impurities, $\tau^{\text {alloy }}(x)$ is the alloy scattering time, which explicitly depends on the disorder level $x$.

Most recently, ideas were put forth according to which at these filling factors disorder may induce microscopic puddles $52 / 58$. In Ref ${ }^{[52}$, in the presence of a long range scattering due to remote ionized dopants the electron gas breaks up into puddles of compressible and incompressible regions ${ }^{53}$ and the measured energy gap is determined by a so-called saddle-point energy gap originating from thermally driven tunneling between the puddles 52 . In this mode ${ }^{52}$ an inflection point in the Arrhenius plots of $\ln \left(R_{x x}\right)$ versus $1 / T$ needs to be established. However, such a data analysis is fraught with difficulties because of a commonly occuring measurement artifact. Indeed, this inflection point is expected to occur at low temperatures at which electrons may not necessarily thermalize to the refrigerator temperature, unless special thermalization techniques are used 37 . When electrons do not fully thermalize, an inflection in the Arrhenius plots is observed which, however, is not necessarily related to the puddling effect decribed in the model. In addition, it is not clear whether this model is applicable for short range scattering potentials, such as the alloy potential in our samples. Motivated by recent thermal Hall conductance measurements 59 , other theoretical work advocates for the formation of competing Pfaffian and antiPfaffian puddles on the microscopic scale $e^{54} \sqrt[58]{6}$. Within these models, the temperature dependence of the magnetoresistance and its relationship to the energery gap have not yet been worked out.

In conclusion, we have examined the energy gaps of the even denominator fractional quantum Hall states in a series of alloy samples. Energy gaps in a series of alloy disorder samples at both $\nu=5 / 2$ and $\nu=7 / 2$ are supressed similarly with alloy disorder. In order to separate disorder and other effects, we used an analysis of energy gaps proposed by Morf and d'Ambrumenil. We found that the dimensionless intrinsic gaps are consistent with numerical results, but are larger than those obtained from pristine samples of similar density published in the literature. Furthermore, the disorder parameter exhibited significant scatter. However, a modification of the model enabled by a shared intrinsic gap yielded much improved results. We found that the disorder broadening parameter may be split into contributions from longrange scattering due to remote ionized impurities and that of short-range scattering due to alloy disorder. The latter was found to increase linearly on the alloy content of our samples.

This work was supported by the DOE BES Experimental Condensed Matter Physics program under the Award No. DE-SC0006671.
1 D.C. Tsui, H.L. Stormer, and A.C. Gossard, Phys. Rev. Lett. 48, 1559 (1982).

2 W. Pan, H.L. Stormer, D.C. Tsui, L.N. Pfeiffer, K.W. Baldwin, and K.W. West, Phys. Rev. Lett. 88, 176802 (2002).

3 J.S. Xia, W. Pan, C.L. Vicente, E.D. Adams, N.S. Sullivan, H.L. Stormer, D.C. Tsui, L.N. Pfeiffer, K.W. Baldwin, and K.W. West, Phys. Rev. Lett. 93, 176809 (2004).

${ }^{4}$ G.S. Boebinger, H.L. Stormer, D.C. Tsui, A.M. Chang, J.C.M. Hwang, A.Y. Cho, C.W. Tu, and G. Weimann, Phys. Rev. B 36, 7919 (1987).

${ }^{5}$ R.L. Willett, H.L. Stormer, D.C. Tsui, A.C. Gossard, and J.H. English, Phys. Rev. B 37, 8476 (1988).

${ }^{6}$ R.R. Du, H.L. Stormer, D.C. Tsui, L.N. Pfeiffer, and K.W. West, Phys. Rev. Lett. 70, 2944 (1993).

7 H.C. Manoharan, M. Shayegan, and S.J. Klepper, Phys. Rev. Lett. 73, 3270 (1994).

${ }^{8}$ W. Li, D.R. Luhman, D.C. Tsui, L.N. Pfeiffer, and K.W. West, Phys. Rev. Lett. 105, 076803 (2010).

9 B.-H. Moon, L.W. Engel, D.C. Tsui, L.N. Pfeiffer, and K.W. West, Phys. Rev. B 92, 035121 (2015).

10 X. Fu, Q. Shi, M.A. Zudov, G.C. Gardner, J.D. Watson, and M.J. Manfra, Phys. Rev. B 99, 161402(R) (2019).
11 D.N. Sheng, X. Wan, E.H. Rezayi, K. Yang, R.N. Bhatt, and F. D. M. Haldane, Phys. Rev. Lett. 90, 256802 (2003).

12 X. Wan, D.N. Sheng, E.H. Rezayi, K. Yang, R.N. Bhatt, and F. D. M. Haldane, Phys. Rev. B 72, 075325 (2005).

13 Z. Liu and R.N. Bhatt, Phys. Rev. Lett. 117, 206801 (2016).

14 Z. Liu and R.N. Bhatt, Phys. Rev. B 96, 115111 (2017).

15 B.A. Friedman, Int. J. Mod. Phys. B 32, 1850338 (2018).

16 R.L. Willett, J.P. Eisenstein, H.L. Störmer, D.C. Tsui, A.C. Gossard, and J.H. English, Phys. Rev. Lett. 59, 1776 (1987).

17 J.P. Eisenstein, R.L. Willett, H.L. Stormer, L.N. Pfeiffer, and K.W. West, Surf. Sci. 229, 31 (1990).

18 W. Pan, J.-S. Xia, V. Shvarts, D.E. Adams, H.L. Stormer, D.C. Tsui, L.N. Pfeiffer, K.W. Baldwin, and K.W. West, Phys. Rev. Lett. 83, 3530 (1999).

19 J.P. Eisenstein, K.B. Cooper, L.N. Pfeiffer, and K.W. West, Phys. Rev. Lett. 88, 076801 (2002).

${ }^{20}$ W. Pan, J.S. Xia, H.L. Stormer, D.C. Tsui, C. Vicente, E.D. Adams, N.S. Sullivan, L.N. Pfeiffer, K.W. Baldwin, and K.W. West, Phys. Rev. B. 77, 075307 (2008).

21 H.C. Choi, W. Kang, S. Das Sarma, L.N. Pfeiffer, and K.W. West, Phys. Rev. B. 77, 081301(R) (2008). 
22 C.R. Dean, B.A. Piot, P. Hayden, S. Das Sarma, G. Gervais, L.N. Pfeiffer, and K.W. West, Phys. Rev. Lett. 100, 146803 (2008).

${ }^{23}$ V. Umansky, M. Heiblum, Y. Levinson, J. Smet, J. Nübler, and M. Dolev, J. Cryst. Growth. 311, 1658 (2009).

24 J. Nuebler, V. Umansky, R. Morf, M. Heiblum, K. von Klitzing, and J. Smet, Phys. Rev. B. 81, 035316 (2010).

25 N. Samkharadze, J.D. Watson, G. Gardner, M.J. Manfra, L.N. Pfeiffer, K.W. West, and G.A. Csáthy, Phys. Rev. B. 84, 121305(R) (2011).

26 W. Pan, N. Masuhara, N.S. Sullivan, K.W. Baldwin, K.W. West, L.N. Pfeiffer, and D.C. Tsui, Phys. Rev. Lett. 106, 206806 (2011).

27 G. Gamez and K. Muraki, Phys. Rev. B. 88, 075308 (2013).

28 N. Deng, G.C. Gardner, S. Mondal, E. Kleinbaum, M.J. Manfra, and G.A. Csáthy, Phys. Rev. Lett. 112, 116804 (2014).

29 C. Reichl, J. Chen, S. Baer, C. Rössler, T. Ihn, K. Ensslin, W. Dietsche, and W. Wegscheider, New Journal of Physics 16023014 (2014).

${ }^{30}$ Q. Qian, J. Nakamura, S. Fallahi, G.C. Gardner, J.D. Watson, S. Lüscher, J.A. Folk, G.A. Csáthy, and M.J. Manfra, Phys. Rev. B 96, 035309 (2017).

31 G. Moore and N. Read, Nucl. Phys. B. 360, 362 (1991).

32 C. Nayak, S.H. Simon, A. Stern, M. Freedman, and S. Das Sarma, Rev. Mod. Phys. 80, 1083 (2008).

33 A. Stern, Nature (London) 464, 187 (2010).

${ }^{34}$ R. Morf and N. d'Ambrumenil, Phys. Rev. B. 68, 113309 (2003).

35 W. Li, G.A. Csáthy, D.C. Tsui, L.N. Pfeiffer, and K.W. West, Appl. Phys. Lett. 83, 2832 (2003).

36 G.C. Gardner, J.D. Watson, S. Mondal, N. Deng, G.A. Csáthy, and M.J. Manfra, Appl. Phys. Lett. 102, 252103 (2013).

37 N. Samkharadze, A. Kumar, M.J. Manfra, L.N. Pfeiffer, K.W. West, and G.A. Csáthy, Rev. Sci. Instrum. 82, 053902 (2011).

38 E. Kleinbaum, A. Kumar, L.N. Pfeiffer, K.W. West, and G.A. Csáthy, Phys. Rev. Lett. 114, 076801 (2015)
39 N. Deng, A. Kumar, M.J. Manfra, L.N. Pfeiffer, K.W. West, and G.A. Csáthy, Phys. Rev. Lett. 108, 086803 (2012).

40 K. Park, V. Melik-Alaverdian, N.E. Bonesteel, and J.K. Jain, Phys. Rev. B 58, R10167 (1998).

41 R.H. Morf, N. d'Ambrumenil, and S. Das Sarma, Phys. Rev. B. 66, 075408 (2002).

42 M.R. Peterson, Th. Jolicoeur, and S. Das Sarma, Phys. Rev. B 78, 155308 (2008).

43 M.R. Peterson, Th. Jolicoeur, and S. Das Sarma, Phys. Rev. Lett. 101, 016807 (2008).

44 Z. Papić, N. Regnault, and S. Das Sarma, Phys. Rev. B 80, 201303(R) (2009).

45 K. Pakrouski, M.R. Peterson, Th. Jolicoeur, V.W. Scarola, C. Nayak, and M. Troyer, Phys. Rev. X 5, 021004 (2015).

46 A. Tylan-Tyler and Y. Lyanda-Geller, Phys. Rev. B 91, 205404 (2015).

47 R.H. Morf, Phys. Rev. Lett. 80, 1505 (1998).

48 A. Wójs and J.J. Quinn, Phys. Rev. B 74, 235319 (2006).

49 A. Wójs, C. Tőke, and J.K. Jain, Phys. Rev. Lett. 105, $096802(2010)$.

50 A. Kumar, G.A. Csáthy, M.J. Manfra, L.N. Pfeiffer, and K.W. West, Phys. Rev. Lett. 105, 246808 (2010).

51 A.E. Feiguin, E. Rezayi, C. Nayak, and S. Das Sarma, Phys. Rev. Lett. 100, 166803 (2008).

52 N. d'Ambrumenil, B.I. Halperin, and R.H. Morf, Phys. Rev. Lett. 106, 126804 (2011).

53 D.G. Polyakov and B.I. Shklovskii, Phys. Rev. Lett. 74, 150 (1995).

54 X. Wan and K. Yang, Phys. Rev. B 93, 201303(R) (2016).

55 D.F. Mross, Y. Oreg, A. Stern, G. Margalit, and M.Heiblum, Phys. Rev. Lett. 121, 026801 (2018).

56 C. Wang, A. Vishwanath, and B. I. Halperin, Phys. Rev. B 98, 045112 (2018).

57 B. Lian and J. Wang, Phys. Rev. B 97, 165124 (2018).

58 W. Zhu and D.N. Sheng, Phys. Rev. Lett. 123, 056804 (2019).

59 M. Banerjee, M. Heiblum, V. Umansky, D.E. Feldman, Y. Oreg, and A. Stern, Nature (London) 559, 205 (2018). 